\title{
Systemic Treatment in Bone Metastases
}

\author{
Oğuzhan KESEN, (D) Timuçin ÇiL
}

Department of Medical Oncology, University of Health Sciences, Adana City Training and Research Hospital Faculty of Medicine, Adana-Turkey

\section{Introduction}

Skeletal-related events in cancer patients, including both bone loss and bone metastases caused due to cancer treatment, are a common relapse site for many solid organ cancers, especially such as lung, breast, and prostate. Bisphosphonates and other systemic treatments that inhibit osteoclast activity may prevent, reduce, or delay such cancer-related and treatment-related skeletal complications in patients with both early and advanced cancer.[1]

\section{Bone Metastases}

There is a significant risk in cancer patients with metastatic disease for skeletal complications, including bone metastases and bone loss, which are generally associated with treatment. Bone metastases are associated with significant morbidity in cancer patients. Skeletal complications of bone metastases are often referred to as skeletal-related events (SRE) that may cause a fracture, hypercalcemia, loss of skeletal integrity, spinal cord compression. Patients with such SREs, especially the ones with symptomatic bone metastasis may require surgery, radiotherapy, and systemic treatment. Bone metastases are also associated with pain while movement, which significantly affects the quality of life (QOL). SREs are also common among patients with metastatic bone disease and especially in the ones with lytic metastases. As per previous reports, before routine use of bisphosphonates, SRE is developed in more than 50 percent of patients treated for metastatic breast cancer.[2] Also, it has been reported that among the breast cancer patients with lytic metastases without osteoclast inhibition, SRE has been developed on an average for every three to four months. [3]

\section{Bone Loss}

Among cancer patients with treatment-related bone loss, various risk factors include early ovarian failure due to chemotherapy, the use of aromatase inhibitors for breast cancer, androgen deprivation therapy for prostate cancer, and the use of glucocorticoids. In addition, as in the general population, various non-oncological factors may increase the risk of osteoporosis or osteoporosis-related fractures. These include smoking, excessive alcohol intake, reduced exercise, low calcium intake, vitamin $\mathrm{D}$ deficiency, genetic history and pharmacological agents such as proton pump inhibitors, anticonvulsants, anticoagulants and certain antidepressants.[1]

\section{Biphosphonates}

Bisphosphonates are an integral component of cancer therapy in metastatic cancer patients with bone metastasis. This treatment choices reduces the complications of metastatic bone disease, mainly by reducing the prevalence of SREs.[1,4] Moreover, bisphosphonates are used in the prevention and treatment of both treatment-related and non-treatment-related bone loss. Additionally, bisphosphonates are used in the acute treatment of cancer-related hypercalcemia and in maintenance therapy to reduce the risk of developing hypercalcemia.

\section{The Mechanism of Action of Bisphosphonates}

Bisphosphonates are analogs of pyrophosphate. The side chains of the central carbon form R1 and R2. These side-chain sites determine the affinity and strength of different bisphosphonates for hydroxyapatite. It was previously assumed that bisphosphonates prevent bone

\section{Dr. Timuçin ÇíL}

Sağlık Bilimleri Üniversitesi,

Adana Şehir Eğitim ve Araştırma Hastanesi Tıp Fakültesi,

Tıbbi Onkoloji Bilim Dalı,

Adana-Turkey

E-mail:drtimucincil@gmail.com 
loss by preventing the dissolution of hydroxyapatite.[5] However, later studies reported that bisphosphonates instead reduce bone resorption and increase mineralization by inhibiting osteoclast activity. $[1,5]$

There are two types of bisphosphonates that have slightly different effects in killing osteoclast cells, namely, nitrogen-free and nitrogen-containing. Nitrogen-containing bisphosphonates are stronger osteoclast inhibitors that include pamidronate, alendronate, ibandronate, risedronate, and zoledronic acid. Nitrogenfree bisphosphonates include etidronate, clodronate, and tiludronate. Bisphosphonates have a apoptotic effect on osteoclasts, affecting their differentiation and maturation and thus act as active bone resorption inhibitors. Bisphosphonates have also been shown to affect macrophages, gamma delta $\mathrm{T}$ cells, osteoblasts, and tumor cells in prephase models. In addition to the effects of bisphosphonates on osteoclast inhibition, they might also have anti-tumor and/or anti-angiogenic effects; however, this remains controversial. There are further studies in progress to define clinically relevant effects of bisphosphonates in cancer patients in a better way.[6,7]

\section{Side Effects}

Bisphosphonates treatment is well tolerated in most cases. However, the best known complications include acute allergic reactions, ocular inflammation, renal failure, electrolyte imbalance (especially hypocalcemia), osteonecrosis of the jaw (ONJ), rarely bone joint or muscle pain, and atrial fibrillation. The nephrotoxicity of bisphosphonates is a common events. it's two mechanism for this. These are dose-dependent and infusion time-dependent. Renal toxicity can be decreased by observing recommended infusion time, optimizing hydration before bisphosphonate therapy (especially in patients with Bence-Jones proteinuria), and avoiding concurrent nephrotoxic drugs. An initial lower dose of zoledronic acid is recommended for patients with pre-existing renal impairment. Serum creatinine should be checked prior to each dose in all the patients with metastatic disease who are receiving IV bisphosphonates. Dose reduction is required in patients with below $60 \mathrm{~mL} / \mathrm{min}$. The recommended doses in patients with creatinine clearance levels, $50-60 \mathrm{~mL} / \mathrm{min}$; $40-49 \mathrm{~mL} / \mathrm{min}$; and $30-39 \mathrm{~mL} / \mathrm{min}$ should be $3.5 \mathrm{mg}$; $3.3 \mathrm{mg}$, and $3 \mathrm{mg}$, respectively. In addition, patients receiving bisphosphonates for multiple myeloma should also have periodic monitoring for albuminuria. Osteonecrosis of the jaw (ONJ) is a rare complication of chronic treatment with intravenous high-density bis- phosphonates in cancer patients that are being increasingly recognized nowadays. It typically results in pain, infection, and necrotic bone tissue in the mandible or maxilla. Risk factors include tooth extraction, poor dental hygiene, poorly fitting dentures, and advanced periodontal disease. Due to the difficulty involved in the treatment of ONJ, prevention is especially emphasized. All patients should be examined by a dentist and, if possible, their dental treatment should be done before starting ONJ treatment, including optimization of periodontal health and removal of unrecoverable teeth. During therapy, all patients should be informed about daily brushing, flossing, and the use of antibacterial mouthwashes, along with recommended dental examinations, dentures, and routine cleaning. Also, patients, in turn, should inform their dentists that whether they are taking any agents for bone metastasis so that invasive dental procedures and especially extraction can be avoided while receiving active treatment. Also, during treatment, oncologists should carefully examine the oral hygiene status and monitor it closely. Although, the patients who develop ONJ while receiving the bisphosphonate treatment are recommended to discontinue its use immediately. Although effective inhibition of osteoclast activity can cause hypocalcemia and hypophosphatemia, the former does not occur in most patients due to compensating mechanisms. Patients with kidney failure or vitamin $\mathrm{D}$ deficiency are at higher risk for electrolyte imbalance and therefore, before starting treatment with bisphosphonates, the levels of Calcium and vitamin D levels should be evaluated and corrected if low. During the treatment, the levels of serum calcium, magnesium, and phosphate, as well as vitamin D should also be monitored periodically. If there are no contraindications, then patients receiving bisphosphonates should receive calcium and vitamin D supplements to reduce the risk of bisphosphonate-induced hypocalcemia and to maintain bone health. Since oral calcium absorption is regulated by parathyroid hormone, which decreases with the increase in serum calcium levels, there is no harm in taking calcium and vitamin D for patients who are at risk of hypercalcemia in the case of bone metastases.

\section{Activity \\ Breast cancer}

Metastatic breast cancer patients with bone metastasis should be used anti-resorptive treatment. Bisphosphonate therapy may widely used for this approach and prevent and delay skeletal complications and relieve bone pain. However overall survival data is not suffient 
yet. Metastatic breast cancer patients with bone metastasis and non clinical bone metastases, the treatment with bisphosphonate is not effective to reduce skeletalrelated events. Consequently, it is recommended that treatment with high-dose bisphosphonates could be initiated after bone metastases have been identified, unless part of a clinical trial. In women with breast cancer who are receiving chemotherapy or aromatase inhibit the bisphosphonates therapy may also prevent treatment-related bone loss. It has also been shown that changing the treatment for every three months after monthly use in 12-15 months in patients with breast cancer does not increase the development of SRE but instead reduces the side effect profile.

\section{Multiple myeloma}

Intravenous bisphosphonate therapy is used for patients with multiple myeloma or having any of the below:

- Demonstration of bone or spine compression fracture from osteopenia on plain radiography or imaging techniques.

- Demonstration of low bone mineral density without lytic bone destruction.

- Osteolytic disease with symptom (especially pain).

- In addition to radiotherapy, analgesics, or surgery to stabilize fractures or reduce fracture risks.

\section{Lung cancer and other solid tumors}

Clinical trial data on bisphosphonates in lung cancer and other solid tumors are more limited as compared to data available for multiple myeloma, breast, and prostate cancer. In a placebo-controlled study with a population of 773 patients with skeletal metastases from non-breast and non-prostate cancers (including non-small cell and small cell lung, renal cell, thyroid, and head and neck cancers), a significant reduction in the number of SREs was found in patients who were treated with zoledronic acid (47\%) versus without the treatment (38\%) and took a significantly longer time for the first event (163 versus 230 days). [8] These benefits continued in long-term treatment and during the follow-up time of approximately 21 months.[9] Additional studies supporting the use of bisphosphonates in lung cancer have also been shown in a previous study comparing 365 patients with bone metastasis treated with zoledronic acid with 2174 patients without zoledronic acid.[10] A significant reduction of skeletal complications was observed in patients receiving bisphosphonates. Based on these results, zoledronic acid was recommended for use in selected patients with metastatic lung cancer, renal cell carcinoma, and other solid tumors with evidence of bone metastasis. [1,4,11] Patients should be selected according to their expected survival time and general palliative benefit expectation.[12,13] In addition to their benefits in reducing the rate of SREs, bisphosphonates could also reduce bone pain from skeletal metastases and improve quality of life, a finding that is best demonstrated in patients with metastatic thyroid cancer. The optimum duration of bisphosphonate therapy is controversial. Continuation of treatment is recommended to minimize the frequency of SREs and/or delay in their development.

\section{Prostate cancer}

Prostat cancer is the most frequent cancer type. $80 \%$ of patients have bone metastasis. Bisphosphonates are not recommended in patients with castration susceptibility, except the ones with acute complications such as hypercalcemia and pathological fracture. It has been shown that it does not reduce the development of new bone metastases and SREs in castration-sensitive disease. Bisphosphonates greatly minimize the development of the first skeletal-related case in castration-resistant disorder but result in an increase in pain score and a substantial decline in analgesic use. Using an example from breast cancer research, the use of bisphosphonate treatment in prostate cancer patients may be brought to the forefront every three months.

\section{Denosumab}

In addition to bisphosphonates, osteoclast inhibition can also be achieved by targeting the receptor activator of the nuclear factor kappa B ligand (RANKL), which is a key component in the pathway of osteoclast formation and activation. RANKL expression in the bone is also thought to lead to the development of bone metastatistics by binding on the surface of tumor cells with the bone receptor activator kappa B (RANK). Denosumab, a monoclonal antibody targeting RANKL, is effective in the treatment of postmenopausal osteoporosis as well as in breast and prostate cancer patients who are at risk of bone deterioration due to cancer therapy or endocrine therapy with aromatase inhibitors or androgen deficiency therapy. In phase III studies, denosumab significantly prolonged the time to first skeletal-related event (SRE) compared to zoledronic acid among patients with bone metastases in breast and prostate cancer. It has also been studied in a variety of other malignancies.[14,15] Earlier, denosumab and zoledronic acid were compared in a phase III study among 1776 
patients with bone metastases caused by solid tumors other than breast or prostate cancer or diagnosed with multiple myeloma ( $40 \%$ non-small cell lung cancer, $10 \%$ multiple myeloma, 9\% renal cell carcinoma, $6 \%$ small cell lung cancer, 5 percent other tumor types). [15] Denosumab demonstrated a significant difference compared to zoledronic acid in delaying the time to the first skeletal-related event (SRE, 16.3 vs. 20.6 months, HR 0.84, 095\% CI 0.71-0.98, $\mathrm{p}=0.03$ ). However, when multivariate analysis was performed to test for superiority, the difference was not statistically significant $(p=0.06)$. Considering the secondary endpoints, it was found that denosumab reduced radiotherapy exposure to the bone by 22 percent compared to zoledronic acid ( $\mathrm{p}=0.026)$, preventing worsening of pain and its intervention. The Brief Pain Inventory score has been shown to significantly reduce the frequency of switching from weak opioid use to strong opioids at 3 months compared to zoledronic acid $(\mathrm{p}<0.05)$.[16] However, in the analysis of 811 patients with lung cancer (including 702 non-small cell carcinomas [NSCLC] and 109 with small cell carcinomas [SCLC]), treatment with denosumab generally provided a small but statistically significant survival advantage (median 7.7 versus 8.9 months, HR 0.80, 95\% CI 0.67-0.95).[17] A similar survival benefit trend was observed in both NSCLC and SCLC patients. In patients with multiple myeloma, mortality tended to be higher with denosumab compared to zoledronic acid (HR for death 2.26, 95\% CI 1.13-4.50), but the number of patients in this population was small.[15] A patient-level metaanalysis of phase III trials comparing zoledronic acid to denosumab for metastatic bone disease in the breast, prostate, or other solid tumors found that denosumab was superior to zoledronic acid in terms of risk reduction. $[15,18,19]$ The study of Lipton et al.[20] concluded that denosumab was superior to zoledronic acid in reducing the risk of the first on-study SRE (HR 0.83, 95\% CI 0.76-0.90). Also, denosumab significantly delayed the time to first SRE or malignant hypercalcaemia in comparison with zoledronic acid (median 19.4 vs. 26.6 months). Overall survival and disease progression were similar with both treatments. Unlike zoledronic acid, denosumab did not require monitoring or dose modification, or cessation of therapy for renal failure and was not associated with acute allergic reactions. It was found that hypocalcemia was more common with denosumab, and osteonecrosis of the jaw occurred at a similar rate. Based on these data, denosumab is approved for the treatment of patients with bone metastases in solid tumors but without multiple myeloma.
The recommended dose and schedule for denosumab for the prevention of SREs is $120 \mathrm{mg}$ administered subcutaneously every four weeks.

\section{Side Effects}

Osteonecrosis of the jaw (ONJ) and hypocalcemia are notable side effects shared by both denosumab and high potency IV bisphosphonates apart from other electrolyte disturbances. While the risk of $\mathrm{ONJ}$ is similar for denosumab and bisphosphonates, the risk of hypocalcemia is slightly higher in the case of denosumab. There are similar recommendations for the assessment and management of dental health and serial monitoring of electrolytes during treatment who are receiving bisphosphonates. Unlike bisphosphonates, denosumab is not eliminated by the kidneys and therefore does not require monitoring of renal functions or any dose adjustments during treatment. However, while initiating therapy in patients with severe renal impairment who are more prone to hypocalcemia should be closely monitored for any related toxicity. Before starting treatment, the levels of calcium and vitamin D should be evaluated and corrected if low. Considering the absence of any contraindications (e.g., pre-existing hypercalcemia, recurrent kidney stones) in patients who are receiving denosumab, it is recommended to use calcium and vitamin $\mathrm{D}$ supplements to provide adequate calcium for bone repair/healing and prevent secondary hyperparathyroidism and hypocalcemia.

Since parathyroid hormone regulates oral calcium absorption, which reduces as serum calcium increases, using calcium and vitamin D in patients at risk for hypercalcemia due to bone metastases is not a problem. The long-term application of bisphosphonates does not include long-term adverse effects. Although temporary bone pain, fatigue, vomiting, and anaemia can be observed, these side effects are manageable, too.

\section{Conclusion}

There is a significant risk in cancer patients with metastatic disease for skeletal events. These are bone lose and bone metastasis and bone lose is generally associated with treatment. Other associated skeletalrelated events risks include bone metastases, fractures, loss of skeletal integrity requiring surgery or radiotherapy, spinal cord compression, and malignant hypercalcemia. Bisphosphonates and other osteoclast inhibitors such as denosumab are effective and they reduce the morbidity of metastatic bone disease. This agents may 
reduce SREs and are the main systemic treatment options in the emergency treatment and prophylaxis of hypercalcemia. They may also provide palliative benefits for patients with bone pain.

\section{References}

1. Gralow JR, Biermann JS, Farooki A, Fornier MN, Gagel RF, Kumar R, et al. NCCN Task Force Report: Bone health in cancer care. J Natl Compr Canc Netw 2013;11 Suppl 3:S1-50; quiz S51.

2. Domchek SM, Younger J, Finkelstein DM, Seiden MV. Predictors of skeletal complications in patients with metastatic breast carcinoma. Cancer 2000;89(2):363-8.

3. Coleman RE. Uses and abuses of bisphosphonates. Ann Oncol 2000;11 Suppl 3:179-84.

4. Aapro M, Abrahamsson PA, Body JJ, Coleman RE, Colomer R, Costa L, et al. Guidance on the use of bisphosphonates in solid tumours: recommendations of an international expert panel. Ann Oncol 2008;19(3):420-32.

5. Fleisch H. Bisphosphonates: mechanisms of action. Endocr Rev 1998;19(1):80-100.

6. Winter MC, Holen I, Coleman RE. Exploring the anti-tumour activity of bisphosphonates in early breast cancer. Cancer Treat Rev 2008;34(5):453-75.

7. Gnant M, Dubsky P, Fitzal F, Blaha P, Schoppmann S, Steger $\mathrm{G}$, et al. Maintaining bone density in patients undergoing treatment for breast cancer: is there an adjuvant benefit? Clin Breast Cancer 2009;9 Suppl 1:S18-27.

8. Rosen LS, Gordon D, Tchekmedyian S, Yanagihara R, Hirsh V, Krzakowski M, et al. Zoledronic acid versus placebo in the treatment of skeletal metastases in patients with lung cancer and other solid tumors: a phase III, double-blind, randomized trial--the Zoledronic Acid Lung Cancer and Other Solid Tumors Study Group. J Clin Oncol 2003;21(16):3150-7.

9. Rosen LS, Gordon D, Tchekmedyian NS, Yanagihara R, Hirsh V, Krzakowski M, et al. Long-term efficacy and safety of zoledronic acid in the treatment of skeletal metastases in patients with nonsmall cell lung carcinoma and other solid tumors: a randomized, Phase III, double-blind, placebo-controlled trial. Cancer 2004;100(12):2613-21.

10. Hatoum HT, Lin SJ, Smith MR, Barghout V, Lipton A. Zoledronic acid and skeletal complications in patients with solid tumors and bone metastases: analysis of a national medical claims database. Cancer 2008;113(6):1438-45.
11. De Marinis F, Eberhardt W, Harper PG, Sureda BM, Nackaerts K, Soerensen JB, et al. Bisphosphonate use in patients with lung cancer and bone metastases: recommendations of a European expert panel. J Thorac Oncol 2009;4(10):1280-8.

12. Brown JE, Cook RJ, Major P, Lipton A, Saad F, Smith $\mathrm{M}$, et al. Bone turnover markers as predictors of skeletal complications in prostate cancer, lung cancer, and other solid tumors. J Natl Cancer Inst 2005;97(1):59-69.

13. Coleman RE, Major P, Lipton A, Brown JE, Lee KA, Smith $\mathrm{M}$, et al. Predictive value of bone resorption and formation markers in cancer patients with bone metastases receiving the bisphosphonate zoledronic acid. J Clin Oncol 2005;23(22):4925-35.

14. Fizazi K, Lipton A, MarietteX, Body JJ, Rahim Y, Gralow $\mathrm{JR}$, et al. Randomized phase II trial of denosumab in patients with bone metastases from prostate cancer, breast cancer, or other neoplasms after intravenous bisphosphonates. J Clin Oncol 2009;27(10):1564-71.

15. Henry DH, Costa L, Goldwasser F, Hirsh V, Hungria V, Prausova J, et al. Randomized, double-blind study of denosumab versus zoledronic acid in the treatment of bone metastases in patients with advanced cancer (excluding breast and prostate cancer) or multiple myeloma. J Clin Oncol 2011;29(9):1125-32.

16. Vadhan-Raj S, von Moos R, Fallowfield LJ, Patrick DL, Goldwasser F, Cleeland CS, et al. Clinical benefit in patients with metastatic bone disease: results of a phase 3 study of denosumab versus zoledronic acid. Ann Oncol 2012;23(12):3045-51.

17. Scagliotti GV, Hirsh V, Siena S, Henry DH, Woll PJ, Manegold C, et al. Overall survival improvement in patients with lung cancer and bone metastases treated with denosumab versus zoledronic acid: subgroup analysis from a randomized phase 3 study. J Thorac Oncol 2012;7(12):1823-29.

18. Fizazi K, Carducci M, Smith M, Damião R, Brown J, Karsh L, et al. Denosumab versus zoledronic acid for treatment of bone metastases in men with castrationresistant prostate cancer: a randomised, double-blind study. Lancet 2011;377(9768):813-22.

19. Stopeck AT, Lipton A, Body JJ, Steger GG, Tonkin K, de Boer RH, et al. Denosumab compared with zoledronic acid for the treatment of bone metastases in patients with advanced breast cancer: a randomized, double-blind study. J Clin Oncol 2010;28(35):5132-9.

20. Lipton A, Fizazi K, Stopeck AT, Henry DH, Brown JE, Yardley DA, et al. Superiority of denosumab to zoledronic acid for prevention of skeletal-related events: a combined analysis of 3 pivotal, randomised, phase 3 trials. Eur J Cancer 2012;48(16):3082-92. 